\title{
Low Grade Salivary Gland Carcinoma
}

National Cancer Institute

\section{Source}

National Cancer Institute. Low Grade Salivary Gland Carcinoma. NCI Thesaurus. Code C8012.

A salivary gland carcinoma with low grade histopathologic features. It includes the salivary gland polymorphous low grade adenocarcinoma, salivary gland low grade cribriform cystadenocarcinoma, and low grade salivary gland mucoepidermoid carcinoma. It usually follows a non-aggressive clinical course. 